\title{
Optimized gyrosynchrotron algorithms and fast codes
}

\author{
Alexey A. Kuznetsov ${ }^{1,2}$ and Gregory D. Fleishman ${ }^{3,4}$ \\ ${ }^{1}$ Armagh Observatory, Armagh BT61 9DG, Northern Ireland, UK \\ email: aku@arm.ac.uk \\ ${ }^{2}$ Institute of Solar-Terrestrial Physics, Irkutsk 664033, Russia \\ ${ }^{3}$ New Jersey Institute of Technology, Newark, NJ 07102, USA \\ email: gfleishm@njit.edu \\ ${ }^{4}$ Ioffe Physico-Technical Institute, St. Petersburg 194021, Russia
}

\begin{abstract}
Gyrosynchrotron (GS) emission of charged particles spiraling in magnetic fields plays an exceptionally important role in astrophysics. In particular, this mechanism makes a dominant contribution to the continuum solar and stellar radio emissions. However, the available exact equations describing the emission process are extremely slow computationally, thus limiting the diagnostic capabilities of radio observations. In this work, we present approximate GS codes capable of fast calculating the emission from anisotropic electron distributions. The computation time is reduced by several orders of magnitude compared with the exact formulae, while the computation error remains within a few percent. The codes are implemented as the executable modules callable from IDL; they are made available for users via web sites.
\end{abstract}

Keywords. radiation mechanisms: nonthermal, methods: numerical, Sun: flares, Sun: radio radiation, stars: flare, radio continuum: general

Gyrosynchrotron (GS) radiation (incoherent radiation of mildly relativistic electrons spiraling in a magnetic field) makes a dominant contribution into the radio and microwave emission of solar and stellar flares. Therefore, radio observations potentially can be used to diagnose the parameters of the emission sources (such as energy and pitch-angle distributions of the energetic electrons, magnetic field, and plasma density). The diagnostics can be made, e.g., by using the forward-fitting methods (e.g., Fleishman et al. 2009), which requires fast and accurate methods of calculating the GS emission.

The exact formulae for the gyromagnetic radiation are known for several decades (Eidman 1958, 1959; Melrose 1968; Ramaty 1969). However, these formulae are computationally slow, especially when high harmonics of the cyclotron frequency are involved (i.e., in a relatively weak magnetic field). A number of simplified approaches has been proposed (e.g., Petrosian 1981; Dulk \& Marsh 1982; Klein 1987), but those approximations were developed for the isotropic electron distributions only, while real distributions in the solar and stellar flares are often expected to be highly anisotropic. Thus, there is a need to develop algorithms and computer codes that would be fast, accurate, and applicable to both isotropic and anisotropic electron distributions.

In developing such algorithms, we follow the approximation proposed by Petrosian (1981) and Klein (1987), which has been proved to provide a reasonable accuracy in a high-frequency range in the case of the isotropic or weakly anisotropic electron distributions. We have found that, after a few improvements (involving a wider use of numerical methods), this approximation can be extended to the strongly anisotropic distributions, provided that the pitch-angle distribution function is continuous together with its first and second derivatives. The key element of this new algorithm (which we call "continu- 

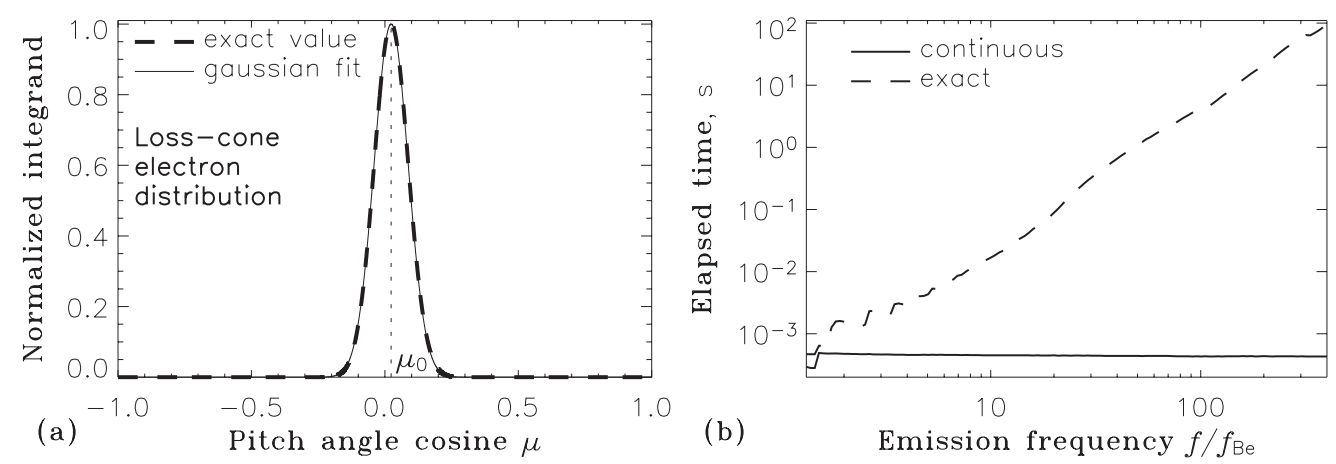

Figure 1. (a) Example of the angular integrand (dashed line) and its gaussian fit (solid line). (b) Time required to calculate the intensity and polarization of the GS emission at a given frequency (a $2 \mathrm{GHz}$ Intel Pentium processor was used for the calculations).

ous") is a possibility to approximate the angular integrand of the exact GS equations by a Gaussian fit, see Fig. 1a, which is then integrated analytically saving the computation time significantly (see Fig. 1b). The comprehensive description of the new approximation is given in the recently published article of Fleishman \& Kuznetsov (2010). Besides a wider applicability range, the new approximation improves the accuracy for the isotropic distributions in comparison with the original Petrosian-Klein algorithm.

Figure 2 demonstrates an example of the GS emission calculated using the exact formulae and new continuous algorithm. The energetic electrons are assumed to have a power-law distribution over energy and a loss-cone distribution over pitch-angle. One can see that the contunuous algorithm provides very high accuracy, especially at high frequencies. The degree of polarization is reproduced with high accuracy. Spectral index of the emission in the optically thin range is reproduced very well, too. At low frequencies, the continuous algorithm is unable to reproduce the harmonic structure of the GS emission; however, the mean level of the spectrum is reproduced well. In Figs. 2a-2c, an additional line shows the corresponding parameters calculated for the isotropic electron distribution. One can see that the considered anisotropy affects significantly the emission intensity, polarization, and spectral index. Nevertheless, the continuous algorithm works excellently even for this (strongly anisotropic) distribution.

Being that accurate, the continuous algorithm is much faster than the exact expressions. As one can notice from Fig. 1b, computation time for the exact formulae grows exponentially with the harmonic number, while for the continuous algorithm this time is nearly constant. As a result, at high frequencies (i.e., where the continuous algorithm is very accurate), the computation time can be reduced by several orders of magnitude in comparison with the exact formulae. In addition to this continuous algorithm, Fleishman \& Kuznetsov (2010) developed a number of codes, which can be gradually tuned to optimize either computation time or accuracy including recovery of the low-frequency harmonic structure of the GS radiation, remaining much faster than the exact codes.

The new algorithms are implemented as executable modules (Windows dynamic link libraries and Linux shared objects) callable from IDL. The codes are called using the IDL call_external function. This approach combines high computation speed with the IDL visualization capabilities. A factorized form of the electron distribution function is adopted: $F(E, \alpha)=u(E) g(\alpha)$, where $E$ and $\alpha$ are the electron energy and pitchangle, respectively. Currently, the code contains 9 built-in energy distributions (including thermal, power-law, kappa, etc.) and 5 pitch-angle distributions (including isotropic, loss-cone, and beam-like); any combination of the energy and pitch-angle distribution 


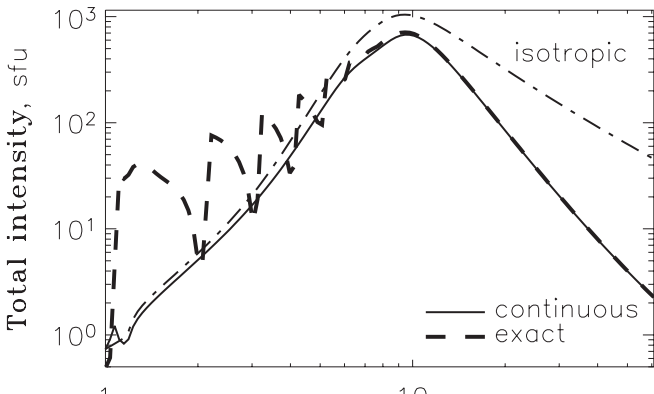

(a)

Emission frequency, $\mathrm{GHz}$

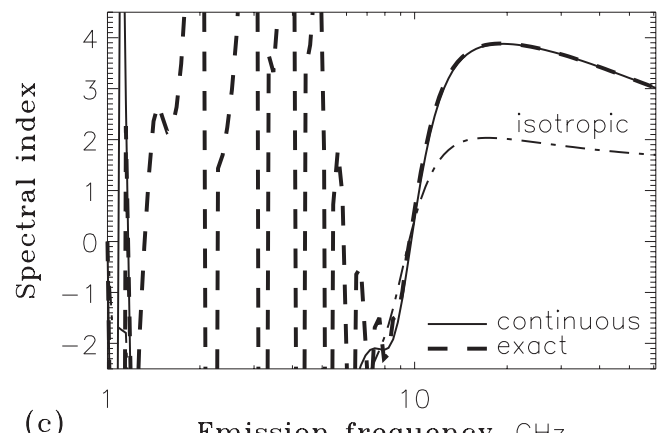

(c)

Emission frequency, $\mathrm{GHz}$

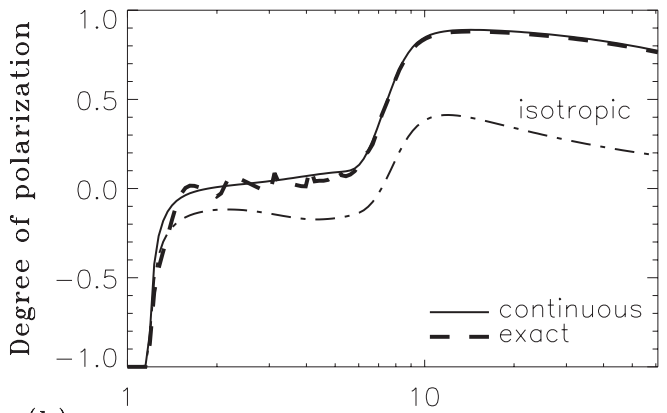

(b)

Emission frequency, $\mathrm{GHz}$

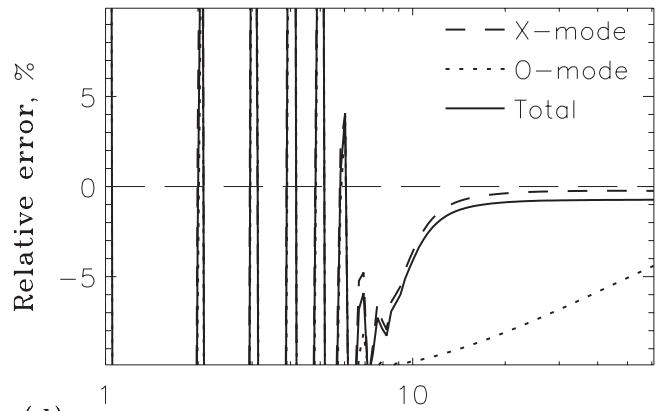

(d)

Emission frequency, $\mathrm{GHz}$

Figure 2. Calculated parameters of the GS emission for the electron distribution of loss-cone type. In the panels (a-c), the dash-dotted line shows the corresponding parameters for the isotropic distribution. Panel (d) shows the relative difference between the emission intensities calculated using the exact and continuous codes.

is possible. The code returns the intensities of the ordinary and extraordinary modes from a homogeneous source located at the Sun (and observed at the Earth), as well as the corresponding gyroabsorption factors which can directly be used for solving the equation of radiation transfer in an inhomogeneous case. Fast GS codes together with the comprehensive documentation are available as the online supplement to the article of Fleishman \& Kuznetsov (2010), and at the web site: star.arm.ac.uk/ ªku/gs/

\section{Acknowledgements}

A.K. thanks the Leverhulme Trust for financial support. This work was supported in part by NSF grants ATM-0707319, AST-0908344, and AGS-0961867 and NASA grant NNX10AF27G to New Jersey Institute of Technology, and by the RFBR grants 08-0292204, 08-02-92228, 09-02-00226, and 09-02-00624.

\section{References}

Dulk, G. A., \& Marsh, K. A. 1982, ApJ, 259, 350

Eidman, V. Y. 1958, Sov. Phys. - JETP, 7, 91

Eidman, V. Y. 1959, Sov. Phys. - JETP, 9, 147

Fleishman, G. D., Nita, G. M., \& Gary, D. E. 2009, ApJ, 698, L183

Fleishman, G. D., \& Kuznetsov, A. A. 2010, ApJ, 721, 1127

Klein, K.-L. 1987, $A \& A A, 183,341$

Melrose, D. B. 1968, Ap\&SSS, 2, 171

Petrosian, V. 1981, ApJ, 251, 727

Ramaty, R. 1969, ApJ, 158, 753 